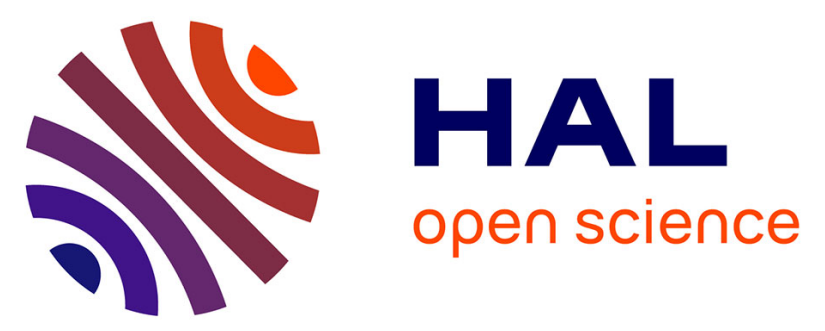

\title{
Temporal dynamics of Kerr frequency combs in whispering-gallery mode resonators
}

Aurélien Coillet, Irina Balakireva, Rémi Henriet, Laurent Larger, Yanne

Kouomou Chembo

\section{- To cite this version:}

Aurélien Coillet, Irina Balakireva, Rémi Henriet, Laurent Larger, Yanne Kouomou Chembo. Temporal dynamics of Kerr frequency combs in whispering-gallery mode resonators. Proceedings 8600 of SPIE Conference Laser Resonators, Microresonators, and Beam Control XV, Jan 2013, United States. pp.860013. hal-00914518

\section{HAL Id: hal-00914518 https://hal.science/hal-00914518}

Submitted on 5 Dec 2013

HAL is a multi-disciplinary open access archive for the deposit and dissemination of scientific research documents, whether they are published or not. The documents may come from teaching and research institutions in France or abroad, or from public or private research centers.
L'archive ouverte pluridisciplinaire HAL, est destinée au dépôt et à la diffusion de documents scientifiques de niveau recherche, publiés ou non, émanant des établissements d'enseignement et de recherche français ou étrangers, des laboratoires publics ou privés. 


\title{
Temporal dynamics of Kerr frequency combs in whispering-gallery mode resonators
}

\author{
Aurélien Coillet, Irina Balakireva, Rémi Henriet, Laurent Larger and Yanne Chembo \\ FEMTO-ST Institute, UMR 6174 CNRS - Université de Franche-Comté, 32 avenue de \\ l'observatoire 25044 Besançon Cedex
}

\begin{abstract}
Based on a modal description of the evolution of the mode's amplitude in a whispering-gallery mode resonator, we numerically study the generation of Kerr combs. We show that a stable primary comb appear for pump power slightly above threshold, enabling potential applications in metrology. For high input power however, chaotic signals are observed.
\end{abstract}

Keywords: Kerr frequency combs, Whispering-gallery mode resonators, Nonlinear dynamics

\section{INTRODUCTION}

Optical frequency combs are sets of regularly spaced spectral lines in the optic frequency range. They are usually generated from mode-locked femtosecond laser, since a periodic train of pulses yield such equidistant lines in the spectral domain. Those combs have many applications in time-frequency metrology, spectroscopy, navigation systems, sensing $[1,2]$... A new way of generating these frequency combs has recently been demonstrated and it relies on the optical Kerr effect in ultra-high-Q whispering-gallery mode (WGM) resonators [3]. In such resonators, the resonant light is enhanced and nonlinear effects can occur at low input powers. In particular, four-wave mixing (FWM) allows for the creation and mixing of frequencies under strict laws of energy and momentum conservation. Only frequency lines separated by the free spectral range (FSR) of the resonator can exist, and a Kerr comb is finally created.

The modeling of this process is however quite complex, since many interactions can occur between modes through the $\chi^{(3)}$ susceptibility of the medium. A few works have been published on this topic [4,5], and a recent work using a modal approach has been proposed [6]. In this work, we use this model to perform numerical simulations and study the temporal behavior of one specific mode of the generated frequency comb.

\section{SYSTEM UNDER STUDY}

The typical scheme of comb generator in a WGM resonator is presented in Figure 1. A continuous-wave laser is amplified and coupled to a WGM resonator thanks to a tapered fiber. In a fiber with such a diameter $(\sim 3 \mu \mathrm{m})$, an important fraction of the propagating mode is located in the air, as an evanescent field. By bringing the fiber close to the resonator, light can be coupled inside the WGM resonator. The light at the output of the fiber is monitored either in the spectral domain with a spectrum analyzer, either in the temporal domain with an oscilloscope.

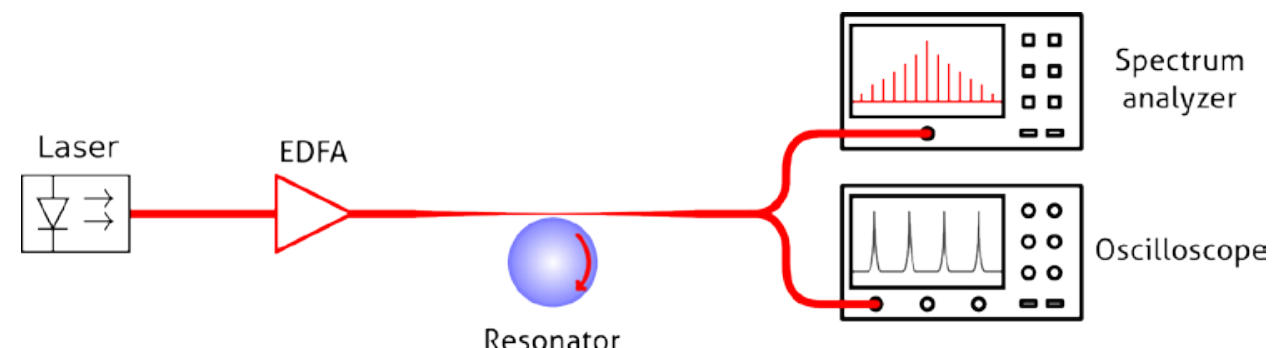

Figure 1. Experimental setup of the Kerr combs generation in a WGM resonator. A continuous laser is amplified and coupled to a WGM resonator using a tapered fiber. The output is either acquired with a spectrum analyzer or an oscilloscope. 
The size of the WGM resonator is chosen so that its FSR is in the wanted frequency range. In our case, we will focus on millimeter-sized resonators for a 10-GHz range FSR. The bulk medium of the resonator can be amorphous (e. g. fused silica) or crystalline. The latter requires a symmetry inversion for the Kerr nonlinearity to be significant. This is the case in $\mathrm{CaF}_{2}$ an $\mathrm{MgF}_{2}$ crystals, which are characterized by very low absorption losses, leading to potential ultra-high Qfactors. Our study takes the example of a 5-mm diameter $\mathrm{CaF}_{2}(\mathrm{n}=1.43$ at $1550 \mathrm{~nm})$ resonator, with a $13.4 \mathrm{GHz}$ FSR and a quality factor of $3.10^{9}$.

\section{MODAL DESCRIPTION}

The modal description proposed in [6] starts from the Maxwell equation and derives the temporal evolution of each resonant mode in the cavity. It takes into account both the FWM gain and the dispersion of the cavity. Using a slowly varying amplitude expansion, the rate equations describing the evolution of the envelope $A_{\eta}$ of each mode is obtained:

$$
\dot{A}_{\eta}=-\frac{1}{2} \Delta \omega_{\eta} A_{\eta}-i g_{0} \sum_{\alpha, \beta, \mu} \Lambda_{\eta}^{\alpha \beta \mu} A_{\alpha} A_{\beta}^{*} A_{\mu} e^{i \varpi_{\alpha \beta \mu \eta} t}+\frac{1}{2} \Delta \omega_{\eta} F_{\eta} e^{i\left(\Omega_{0}-\omega_{\eta}\right) t} .
$$

The modal bandwidth $\Delta \omega_{\eta}$ corresponds to the inverse of the modal photon lifetime. $g_{0}$ stands for the FWM gain while $\Lambda_{\eta}^{\alpha \beta \mu}$ is the intermodal coupling factor between the four interacting modes. The resonator's dispersion is taken into account in the $\varpi_{\alpha \beta \mu \eta}=\omega_{\alpha}-\omega_{\beta}+\omega_{\mu}-\omega_{\eta}$ parameter, since it would be null in the case of perfectly equidistant spectral lines. Finally, the last term corresponds to the external pumping of the cavity, with amplitude $F_{\eta}$ and angular frequency $\Omega_{0}$.
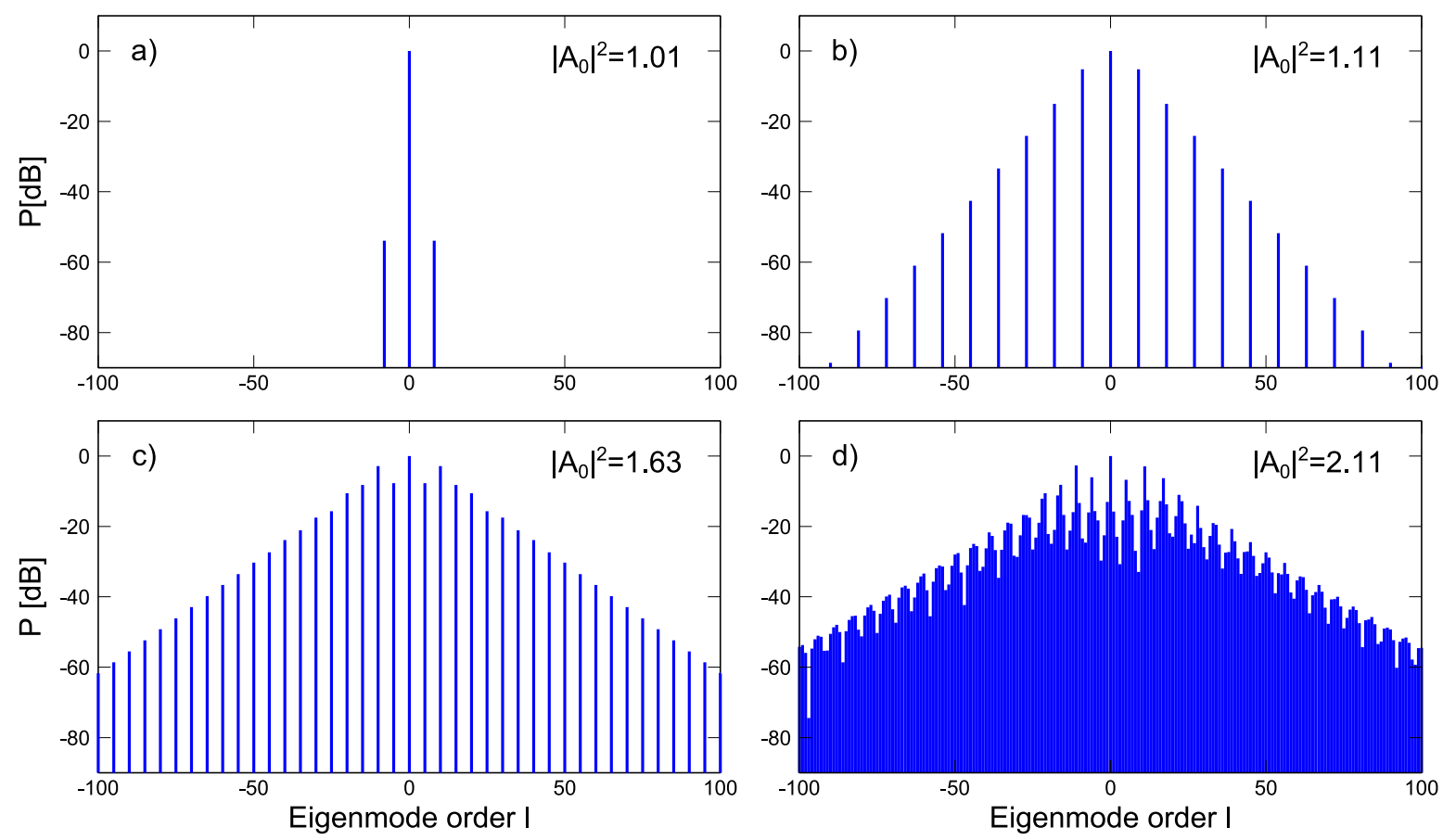

Figure 2. Simulated combs using the modal description for various pumping power. Just above threshold, only a couple of side lines are excited separated from the central line by 9 FSR. At higher excitation, a primary comb is fully formed. When still increasing the pump power, a secondary comb appears, making the interval between adjacent spectral lines half of the one of the primary comb. Finally, at high power, each resonant mode is populated. 
These equations can be numerically solved with a fourth-order Runge-Kutta algorithm. In the following simulations, 201 modes were studied, corresponding to a $\sim 20 \mathrm{~nm}$ wavelength span. Each mode is initialized with a random complex amplitude taken in a Gaussian distribution with mean value and standard deviation equal to 10, corresponding to an average of 100 photons in each mode, since $\left|A_{\eta}\right|^{2}$ is the number of photon in the $\eta^{\text {th }}$ mode. Simulations are performed for a total duration of $2 \mathrm{~ms}$, such that the steady state is reached. The combs displayed in Figure 2 correspond to various pumping amplitude expressed in $\left|A_{0}^{t h}\right|^{2}$ units, where $\left|A_{0}^{t h}\right|^{2}$ is the threshold number of photons in the pumping mode. The parameters used in these simulations are $\Omega_{0}-\omega_{0}=0$ (the pump laser is perfectly resonant), the dispersion term $\varpi_{\alpha \beta \mu \eta}=\frac{1}{2} \zeta\left(l_{\alpha}^{2}-l_{\beta}^{2}+l_{\mu}^{2}-l_{\eta}^{2}\right)$ where $l_{\alpha}^{2}$ corresponds to the $\alpha^{\text {th }}$ mode index and the parameter $\zeta=11800$ rad corresponds to a small, anomalous dispersion. As shown in [6], the presented computer-simulated combs correspond very well to experimental results already obtained.

\section{TEMPORAL EVOLUTION OF EXCITED MODES}

With these simulations, we are now able to study the temporal evolution of each spectral mode separately. In the following, we will focus on the first excited mode in the primary comb, since all if the other modes of the primary comb will behave in the same fashion. With our set of parameters, it correspond to the $8^{\text {th }}, 9^{\text {th }}, 10^{\text {th }}$ or $11^{\text {th }}$ mode depending on the pump power (see [6] for further details). Figure 3 shows this evolution for low excitation power. The y-scale is logarithmic, emphasizing the exponential decrease or growth below and just above threshold. However, for an excitation of $1.69\left|A_{0}^{t h}\right|$, the secondary comb is forming, and the increase is no longer of the exponential type, with a lower rate when the secondary comb appears. Above a certain threshold, the secondary comb acquires energy from the pump, slowing the growth of the primary comb.

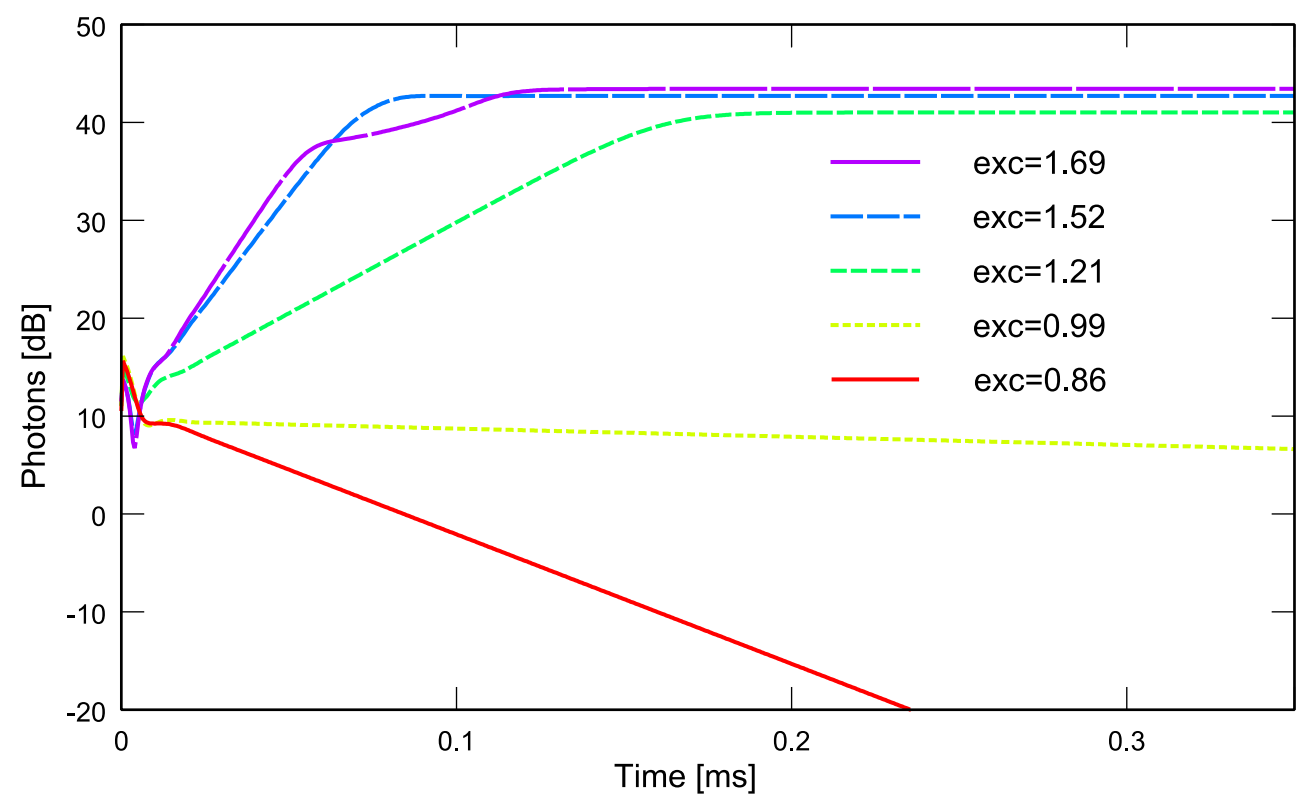

Figure 3. Temporal evolution of the first excited mode for low excitation levels. The y-scale is logarithmic, so that exponential growths appear linear. As expected, the combs start being created for excitation greater than $1\left|A_{0}^{t h}\right|^{2}$. The growth rate and stationary level increase with the excitation power. When the secondary comb is formed (i. e. when the excitation is $1.69\left|A_{0}^{t h}\right|^{2}$ ), the curve shape is modified and energy seems to go from the primary comb to the secondary comb. 
At higher pump power, the behavior becomes more complex, with the apparition of unstable oscillating cycles and finally chaos. Figure 4 present the global evolution of those behaviors, starting with combs just above the threshold and going to pump power as high as 2.11 times the threshold level. While the two first examples do not show any oscillatory behavior, the 1.96|$\left|A_{0}^{t h}\right|^{2}$ curve present attenuated oscillations in the microsecond regime. The last example however, shows a chaotic behavior with fluctuations in the microsecond range too.

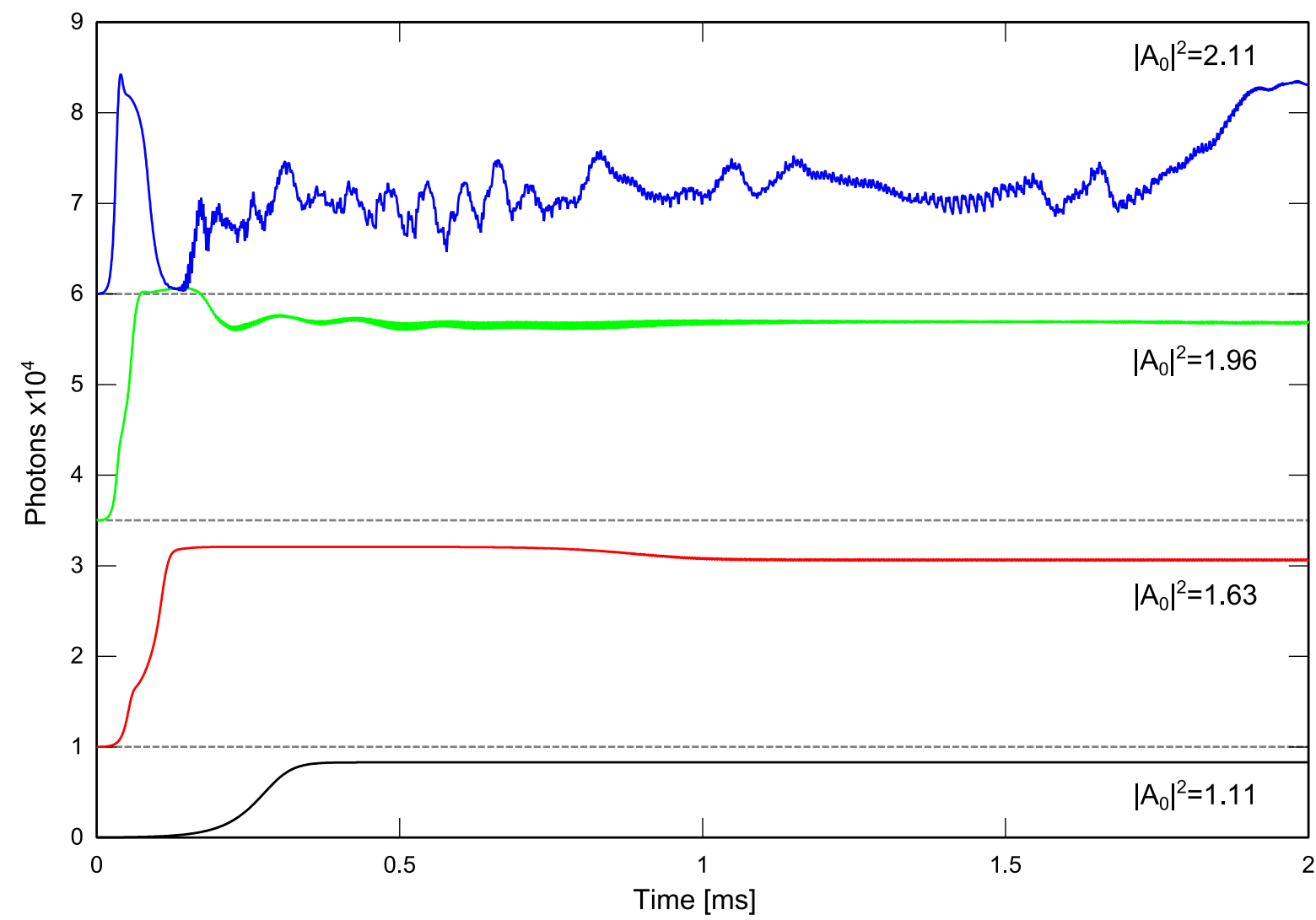

Figure 3. Temporal evolution of the first excited mode for various excitation levels above the threshold level. While the two first cases do not present any oscillations, it is not the case in the third curve, were attenuated oscillations are present. At more than twice the threshold value, the curve clearly shows the chaotic behavior of the combs.

\section{CONCLUSION}

In this work, we studied the temporal evolution of the primary comb occurring at various pump power in a WGM resonator. Using a modal description of the combs, we are able to simulate the evolution of the envelope complex amplitude of each mode. In particular, we show that the growth rate is exponential in the vicinity of the threshold. For greater value of the pump power, the primary comb is slightly perturbed by the apparition of the secondary comb. For higher pump power, the amplitude of the primary comb becomes chaotic.

This study shows that metrological applications could beneficiate from the high stability of the primary comb at low pump power. In this regime, however, the first populated modes are separated by several FSR, depending on the dispersion and pump power.

\section{ACKNOWLEDGMENTS}

Y. C. K. acknowledges financial support from the European Research Council through the project NexPhase (ERC StG 278616). Authors also acknowledge support from the Centre National de la Recherche Spatiale (CNES, France) through 
the project SHYRO (Action R\&T R-S10/LN-0001-004/DA:10076201), drom the ANR project ORA (BLAN 031202) and from the Région Franch-Comté, France.

\section{REFERENCES}

[1] Theodor W. Hänsch, “Nobel Lecture: Passion for precision,” Reviews of Modern Physics 781297 (2006).

[2] Steven T. Cundiff and Jun Ye, "Femtosecond optical frequency combs," Reviews of Modern Physics 75325 (2003).

[3] P. Del'Haye, A. Schliesser, O. Arcizet, T. Wilken, R. Holzwart and T. J. Kippenberg, “Optical frequency comb generation from a monolithic microresonator,” Nature 4501214 (2007).

[4] Imad H. Agha, Yoshitomo Okawachi, Mark A. Foster, Jay E. Sharping, and Alexander L. Gaeta "Four-wavemixing parametric oscillations in dispersion-compensated high-Q silica microspheres,” Phys. Rev. A 76043837 (2007)

[5] Andrey B. Matsko, Anatoliy A. Savchenkov, Dmitry Strekalov, Vladimir S. Ilchenko, and Lute Maleki, "Optical hyperparametric oscillations in a whispering-gallery-mode resonator: Threshold and phase diffusion," Phys. Rev. A 71033804 (2005)

[6] Yanne K. Chembo and Nan Yu, "Modal expansion approach to optical-frequency-comb generation with monolithic whispering-gallery-mode resonators," Physical Review A 82033801 (2010).

[7] Yanne K. Chembo, Dmitry V. Strekalov, and Nan Yu "Spectrum and Dynamics of Optical Frequency Combs Generated with Monolithic Whispering Gallery Mode Resonators,” Phys. Rev. Lett. 104103902 (2010) 